\title{
A SAÚDE ORGANIZACIONAL: ESTUDO DE CAMPO EM UMA INS- TITUIÇÃO DE ENSINO SUPERIOR
}

ORGANIZATIONAL HEALTH: A FIELD STUDY IN A HIGHER EDUCATION INSTITUTION

\section{Taiani Correa da Costa ${ }^{1}$, Luciana Flores Battistella ${ }^{2}$}

RECEBIDO EM: 08/10/2019 | ACEITO EM:03/06/2020

DOI: $10.5902 / 2317175840431$

\section{RESUMO}

Este estudo teve como objetivo identificar a percepção dos Técnicos Administrativos em Educação (TAE) acerca da saúde organizacional em uma instituição pública de ensino superior. Adotou-se a Escala de Percepção de Saúde Organizacional (EPSaO), revalidada por Gomide Jr. e Fernandes (2007), para conduzir uma pesquisa do tipo survey. Posteriormente, foi realizada uma análise fatorial exploratória. A escala EPSaO, apresenta 27 fatores e 2 dimensões, após realizar o procedimento de análise fatorial o modelo encontrado sugeriu 22 variáveis distribuídas em 6 fatores, divergindo, assim, do estudo original. O primeiro fator refere-se à "Integração de equipes"; o segundo diz respeito à "Capacidade de flexibilidade da organização; o terceiro fator "Relação entre pessoas"; o quarto "Cooperação entre pessoas"; o quinto fator "Objetivos organizacionais"; o sexto fator "Valor do trabalho". A versão da EPSaO, resultante desse estudo, sugere a necessidade de maior investigação quanto ao número de fatores para medir a percepção de saúde organizacional.

Palavras-chave: Saúde organizacional; Comportamento organizacional; Serviço Público.

1 Graduada em Direito pela Faculdade de Direito de Santa Maria (2012). Técnica Administrativa em Educação na Universidade Federal de Santa Maria. Mestranda do Programa de Pós-Graduação em Gestão de Organizações Públicas.

2 Professora da Universidade Federal de Santa Maria. Docente nos Programas de Pós-Graduação em Administração Pública (PPGAP) e em Gestão de Organizações Públicas (PPGOP). 


\section{ABSTRACT}

This study aimed to identify the perception of Administrative Technicians in Education about organizational health in a public institution of higher education. We adopted the Organizational Health Perception Scale (EPSaO) revalidated by Gomide Jr. and Fernandes (2007) to conduct a survey-type survey. Subsequently, an exploratory factorial analysis was performed. The EPSaO scale, previously with 27 factors and 2 dimensions, now has 22 variables distributed in the 6 found factors, thus diverging from the original study. The first factor refers to "Team integration"; the second concerns "Flexibility of the organization; the third factor "Relationship between people"; the fourth "Cooperation between people"; the fifth factor "Organizational objectives"; the sixth factor "Labor value". The EPSaO version, resulting from this study, suggests the need for further investigation regarding the number of factors to measure the perception of organizational health.

KEYWORDS: Organizational health; Organizational behavior; Public servisse.

\section{Introdução}

O estudo da saúde organizacional compreende os aspectos relacionados às organizações que podem ocasionar adoecimento das instituições e dos locais de trabalho e, em decorrência disso, dos trabalhadores. As transformações que vêm ocorrendo no mundo do trabalho causadas, especialmente, pela globalização, pelas novas tecnologias e pelas exigências, que o capitalismo impõe às organizações e aos trabalhadores, faz com que aspectos relacionados à saúde física e psicológica desses profissionais estejam em evidência.

Essa realidade também está presente na administração pública, principalmente, após a reforma gerencial do Estado, na qual a lógica que norteia a esfera privada e interesses prioritariamente econômicos encontra-se cada vez mais presentes na vida pública. A reforma, ao enfatizar qualidades do setor privado como a agilidade e a eficiência, contribuiu para deteriorar a imagem dos serviços públicos (RIBEIRO, 2012). No mesmo sentido, Carneiro (p. 25, 2006) complementa que "o servidor público brasileiro possui, portanto, uma imagem estereotipada, com forte apelo negativo, que vem ocasionando perda de prestígio social e gerando baixa estima e consequente adoecimento". Dentre as organizações que sofreram influência direta do arranjo produtivo e organizacional, encontram-se as instituições públicas de ensino superior (IES), que também passaram a ser vistas com olhos gerenciais.

Relevante mencionar que saúde organizacional não deve ser confundida com saúde do trabalhador, são assuntos diferentes apesar de diretamente relacionados, visto que a saúde do trabalhador serve como um indicativo de organização saudável, junto aos critérios de desempenho, efetividade, relações internas e externas, inovação, moral, entre outros. Apesar de não existir um consenso na literatura quanto à definição de saúde organizacional, para 
esse estudo é adotado o conceito de Gomide Junior (1999), tendo em vista que é o autor da escala utilizada nessa pesquisa, definindo-a como a "capacidade da organização de desenvolver altos níveis de adaptabilidade e flexibilidade às demandas internas e externas e ainda desenvolver alto grau de integração entre os empregados e suas equipes de trabalho".

Este trabalho tem como objetivo identificar a percepção dos Técnicos Administrativos em Educação (TAEs) de uma Instituição Pública de Ensino Superior acerca da Saúde Organizacional. A escolha desse público deu-se pela relevância das atividades desempenhadas por esses profissionais, mas que nem sempre são reconhecidos por desenvolverem as atividades-meio nas instituições de ensino, fato que, segundo Loureiro et al. (2018), pode desenvolver sofrimento e afetar a saúde deles. A proposta do estudo justifica-se pela necessidade de aprimorar um instrumento para medir a saúde das organizações que consiga transcrever a complexidade que envolve a matéria, colaborando com a atuação dos gestores, especialmente no que se refere a elaboração e implementação de políticas de gestão de pessoas atinentes aos aspectos que interferem na saúde das instituições e dos colaboradores, além do desenvolvimento de estratégias de intervenção nos ambientes de trabalho.

Quanto aos procedimentos metodológicos caracteriza-se como uma pesquisa quantitativa, de cunho descritivo, realizada em uma Instituição Pública de Ensino Superior do Estado do Rio Grande do Sul, em 2018, que teve a participação de 112 TAEs. Para tanto, adotou-se o modelo de Escala de Percepção de Saúde Organizacional - EPSaO, construída e validada por Gomide Jr., Moura, Cunha e Souza (1999) e revalidada por Gomide Jr. e Fernandes (2008). $\mathrm{O}$ artigo é composto, além dessa introdução, por um referencial teórico, explicação do método utilizado, apresentação e análise dos resultados, considerações finais e referências bibliográficas.

\section{Discussões sobre Saúde Organizacional}

A ideia de saúde organizacional surgiu na década de 1950 centrada na saúde da organização e relacionada à proposta de efetividade, que é definida por Georgopoulos e Tannembaum (1957) como a amplitude em que uma organização alcança seus objetivos sem incapacitar os meios e recursos e sem gerar tensão entre seus membros. Os autores perceberam que os critérios de efetividade organizacional costumavam estar relacionados à produtividade, à lucratividade, à moral, ao comprometimento com a organização, à rotatividade de pessoal, ao absenteísmo e à satisfação dos membros.

Visando esclarecer a confusão acerca da definição de efetividade, Bennis (1962) introduziu critérios para a saúde organizacional como uma extensão do conceito de efetividade. Para isso, realizou a transposição dos critérios de saúde mental dos indivíduos para saúde da organização, estabelecendo como critérios para uma organização saudável a adaptabilidade, a capacidade de 
perceber a realidade e o senso de identidade. Schein (1965) acrescentou o estado de integração como um quarto critério.

Por adaptabilidade, Bennis (1962) entende a "capacidade da organização em resolver problemas que, por sua vez, depende da flexibilidade, entendida como a liberdade de aprender por meio da experiência, de mudar simultaneamente com as condições internas e externas". Já senso de identidade é visto como "conhecimento claro da organização sobre o que ela é, quais são suas metas e onde se pretende chegar". O critério teste da realidade é definido como "capacidade da organização em procurar conhecer, perceber e interpretar corretamente as condições relevantes do ambiente externo e interno para o seu funcionamento".

Por fim, o estado de integração é entendido por Schein (1965) como "o grau de harmonia e integração entre os subsistemas da organização, a fim de que estejam funcionando de forma alinhada à estratégia geral da organização". Para o autor, efetividade é definida como a capacidade da organização sobreviver, de se adaptar, de se manter e crescer, independente das funções desempenhadas. Já Katz e Kahn (1966), relacionam a efetividade ao equilíbrio entre os componentes internos e externos às organizações.

Mello (1978), visando contribuir para elucidar a temática, aponta sete componentes da saúde organizacional: identidade (saber o que é), orientação (conhecer seus objetivos), sensibilidade realista (capacidade de perceber modificações internas e externas), criatividade (criar, inovar, buscar soluções), flexibilidade (capacidade de modificar atitudes, métodos e metas), integração (harmonia, convergência entre as metas da organização e as necessidades dos indivíduos que nela trabalham) e reserva de energia (reservas de recursos).

Após a década de 1990, a noção de saúde organizacional expandiu para além do lucro e da produtividade para incluir o bem-estar dos empregados como um parâmetro importante que afeta a saúde das organizações (JAFFE, 1995). Verifica-se que não há consenso na literatura quanto à definição de saúde organizacional, porém, levando em consideração que um conceito não é mais correto que o outro, é possível referir que existe uma complementaridade entre eles. O Quadro 1 apresenta definições do conceito de saúde organizacional para melhor entender as idiossincrasias trazidas por diferentes autores.

Quadro 1 - Definições de Saúde Organizacional

\begin{tabular}{l|l}
\hline Autor & Definição \\
\hline Cox e Howath (1990) & $\begin{array}{l}\text { Aquela que não gera estresse aos empregados, integração de } \\
\text { conceitos como lucratividade, resultado, controle de qualidade, } \\
\text { absenteísmo, bem-estar físico e psicológico dos empregados, bem } \\
\text { como as atitudes dos trabalhadores para com a organização. }\end{array}$ \\
\hline Jaffe (1995) & $\begin{array}{l}\text { Noção expandida de efetividade organizacional. O conceito deve } \\
\text { considerar as necessidades dos clientes, fornecedores, proprietários } \\
\text { e membros da comunidade. }\end{array}$
\end{tabular}




\begin{tabular}{|l|l|}
\hline Sauter, Lim e Murphy (1996) & $\begin{array}{l}\text { Uma organização saudável é aquela que apresenta baixo estresse, } \\
\text { menor taxa de doença e lesão e valorização do bem-estar } \\
\text { dos funcionários a partir da perspectiva de saúde e segurança } \\
\text { ocupacional. }\end{array}$ \\
\hline Gomide Jr. (1999) & $\begin{array}{l}\text { Capacidade da organização de desenvolver altos níveis de } \\
\text { adaptabilidade e flexibilidade às demandas internas e externas e } \\
\text { ainda desenvolver alto grau de interação entre os empregados e } \\
\text { suas equipes de trabalho. }\end{array}$ \\
\hline Shoaf et al. (2004) & $\begin{array}{l}\text { Noção expandida de efetividade, saúde e bem-estar dos } \\
\text { trabalhadores. }\end{array}$ \\
\hline Wilson et al. (2004) & $\begin{array}{l}\text { É aquela caracterizada por esforços sistemáticos e colaborativos } \\
\text { para maximizar o bem-estar e a produtividade dos funcionários. }\end{array}$ \\
\hline Quick et al. (2007) & $\begin{array}{l}\text { Tem referência em um líder saudável, pois ele é a semente que da } \\
\text { origem à saúde individual e organizacional. }\end{array}$ \\
\hline Fonte: elaborado pelas autoras (2018) &
\end{tabular}

Diante de diferentes entendimentos sobre saúde organizacional, percebe-se a abrangência e a complexidade que envolve o assunto. Shoaf et al. (2004) defende que o conceito de saúde organizacional sofre com a falta de uma abordagem holística que, primeiro, inclua componentes físicos, mentais, organizacionais, relativos ao ambiente de trabalho e suas interações; segundo, promova o equilíbrio desses componentes que precisam funcionar juntos como determinante de um trabalho saudável.

Com a inclusão da saúde e bem-estar do trabalhador como um indicativo de saúde organizacional, observa-se um progresso para os empregados, mas, especialmente, para as organizações. Para Singh e Jha (2017), a saúde de uma organização só pode ser aprimorada por meio da melhoria da saúde e do bem-estar dos trabalhadores que, segundo Dejoy e Wilson (2003), é um processo que deve começar pela própria organização.

Gomide Jr. (2013) relacionou o local de trabalho saudável às políticas de recursos humanos, as quais devem buscar o bem-estar dos indivíduos, sendo imprescindível o conhecimento e a participação dos próprios trabalhadores no processo de formulação. Do mesmo modo, Faria et al. (2017) refere que a melhoria dos processos de trabalho, por meio da participação de todos envolvidos, amplia a saúde institucional e, por consequência, a qualidade dos serviços prestados.

Dejoy e Wilson (2003) destacam temas centrais relacionados à organização de trabalho saudável, aduzindo que se concentram em três domínios: design de trabalho, clima organizacional e futuro do trabalho. Isso envolve mudança organizacional que deve ter o apoio da gerência para ter sucesso; as pessoas percebem e reagem à realidade que experimentam como membros de uma organização; altera a relação empregador-empregado em termos de aumento de oportunidades, troca de informações e envolvimento dos funcionários; a eficácia organizacional é o resultado desejado.

Quick et al. (2007) indicam uma série de fatores influenciadores da saúde organizacional, entre eles fatores contextuais como forças ambientais, glo- 
balização, tecnologia; forças organizacionais como relação de gênero, abusos no local de trabalho, ameaças ambientais; ligação gerencial representada pelo líder saudável que é aquele com integridade e coragem; resultados de saúde subdivididos em saúde individual (presenteísmo da doença e absenteísmo) e saúde organizacional (vigor, vitalidade e produtividade).

Além disso, se os atributos que caracterizam os indivíduos saudáveis fossem empregados no nível organizacional, promoveriam as diferentes categorias de saúde para seus membros. Tais atributos consideram o físico, o psicológico, o espiritual e questões éticas, compreendidos em dimensões que compõem o esquema tridimensional da saúde individual: levando uma vida de propósito, conexão de qualidade para os outros, autoestima positiva e maestria (QUICK et al., 2007).

Já Maclntosh et al. (2007) entendem que conceituar saúde na organização a partir de uma perspectiva de processo é mais apropriado do que uma visão da saúde organizacional como um estado. Para os autores, as atuais conceituações de saúde organizacional podem gerar uma tendência desnecessária a tensões, haja vista que ver a saúde individual como um estado pode limitar indevidamente as expectativas referentes a criar saúde e problemas de saúde dentro dos ambientes organizacionais.

Dessa forma, Maclntosh et al. (2007) contestam a presunção generalizada de que saúde individual e organizacional são positivamente correlacionadas e acreditam ser mais útil discutir "saúde na organização", "que é a saúde como algo criado em processos organizacionais e sociais de interação", no lugar de buscar a relação entre desempenho e resultados organizacionais.

A saúde organizacional se tornou uma questão vital para os empregadores (SINGH; JHA, 2017), que devem administrar a rotina diária do ambiente laboral, a estrutura e o funcionamento das empresas de forma que não causem desgaste entre os indivíduos, transtornos e estresse ocupacional (VISENTINI et al., 2010), além de voltar-se para a necessidade de desempenhos pautados em termos de "responsabilidades financeiras, sociais e ambientais" (HART e COOPER, 2001).

Saúde organizacional é um assunto relativamente menos pesquisado (SINGH; JHA, 2017). Em âmbito nacional, existe poucos estudos disponíveis na academia, pode-se citar os realizados por Gomide Jr. et al. (1999); Gomide Jr. e Nascimento (2008); Visentini et. al. (2010); Fernandes, Gomide Jr. e Oliveira (2011). Assim, percebe-se que o professor Dr. Sinesio Gomide Jr é referência em estudos sobre saúde organizacional no contexto brasileiro. Formado em psicologia, Gomide Jr é professor titular da Universidade Federal de Uberlândia.

Em 1999, Gomide Junior definiu a saúde organizacional como a "capacidade da organização de desenvolver altos níveis de adaptabilidade e flexibilidade às demandas internas e externas e ainda desenvolver alto grau de integração entre os empregados e suas equipes de trabalho". Entretanto, em 2008, Nascimento e Gomide Júnior referem que esse conceito não aponta "a 
efetiva discriminação entre a medida proposta pelos autores e as medidas, já presentes na literatura, que mensuram efetividade das organizações", além de não abrangerem fatores como "qualidade de vida do empregado, absenteísmo e fadiga" (p.3).

Importante mencionar que a única escala validada no Brasil sobre a temática é a Escala de Percepção de Saúde Organizacional (EPSO), construída e validada por Gomide Jr., Moura, Cunha e Souza (1999) e revalidada por Gomide Jr e Fernandes (2008). Em entrevista para a Revista Laborativa, ao ser questionado sobre novos instrumentos editados, Gomide Jr., respondeu que "existe outro instrumento, fruto de uma bolsa de pesquisa. É uma evolução do anterior, mas ainda não foi publicado" (p.70).

\section{Método de estudo}

Trata-se de uma pesquisa descritiva de natureza quantitativa (CRESWELL, 2010), realizada em uma Instituição Pública de Ensino Superior no ano de 2018, com a participação de 112 Técnicos Administrativos em Educação (TAEs). Os participantes foram escolhidos por critério de acessibilidade. Distribuiu-se aproximadamente 200 questionários em diversos setores da instituição, oportunidade em que os servidores foram convidados a participar da pesquisa, assim, os participantes que aceitaram participar preencheram o questionário de forma presencial. Após duas semanas os questionários que tinham sido respondidos foram recolhidos.

O questionário utilizado para a coleta de dados foi dividido em duas partes: a primeira para levantar os fatores relativos à saúde organizacional; a segunda parte buscou identificar o perfil da população investigada, tal como: sexo, faixa etária, escolaridade, tempo de atuação na instituição e se ocupava cargo de chefia.

Para a identificação dos fatores relativos à Saúde Organizacional, adotou-se o modelo de Escala de Percepção de Saúde Organizacional - EPSaO, construída e validada por Gomide Jr., Moura, Cunha e Souza (1999) e revalidada por Gomide Jr. e Fernandes (2008). O modelo é composto por 27 afirmações, divididos em dois fatores: "integração de pessoas e equipes"; e "flexibilidade e adaptabilidade a demandas externas". Todas as dimensões foram medidas por uma escala Likert de 5 pontos (1 - discordo totalmente; 2 - discordo; 3 - nem discordo nem concordo; 4 - concordo; e 5 - concordo totalmente).

A análise dos dados seguiu as seguintes etapas: codificação e preparação dos dados; análise do perfil da amostra; e análise fatorial exploratória da escala. Para isso, foram utilizados os softwares Microsoft Excel e SPSS - Statistical Package for the Social Sciences 18. 


\section{Apresentação e análise dos resultados}

Esta seção encontra-se dividida em duas partes. Inicialmente, são descritos os aspectos relativos a amostra pesquisada. Na segunda parte, apresenta-se a análise fatorial referente ao constructo saúde organizacional.

\subsection{Perfil dos respondentes}

A amostra investigada foi composta por 112 TAEs, dentre os quais $59,8 \%$ $(n=67)$ são do sexo feminino e $40,2 \%(n=45)$ do sexo masculino. Sobre a idade, $35,2 \%(n=38)$ dos respondentes estão na faixa etária de 30 a 39 anos, 31,5\% $(n=34)$ na faixa de 50 a 59 anos e $15,7 \%(n=17)$ com idade de 40 a 49 anos. Os dados provenientes da amostra estratificada demonstram que esta é composta por servidores com idade entre 22 e 70 anos.

Quanto ao nível de instrução, $28,8 \%(n=32)$ possuem cursos de especialização, $27 \%(n=30)$ mestrado, $26,1 \%(n=29)$ superior completo, $8,1 \%(n=6)$ possuem $2^{\circ}$ grau incompleto ou completo e $5,4 \%(n=5)$ doutorado. Em relação ao tempo de serviço junto à instituição, verificou-se maior concentração na faixa de 4 a 9 anos, com 28,7\% ( $n=31$ ), seguido da faixa de até 3 anos com 23,1\% $(n=25)$, sobre os demais tem-se $21,3 \%(n=23)$ com mais de 30 anos, $14,8 \%(n=16)$ com 20 a 29 anos e $12 \%$ ( $n=13)$ entre 10 a 19 anos de trabalho na instituição.

Dentre os participantes da pesquisa, $73,6 \%(n=81)$ afirmaram não possuir cargo de chefia e $26,4 \%(n=29)$ ocupavam cargo de chefia no momento em que responderam o questionário. No que se refere a afastamento por motivo de saúde nos últimos dois anos, $62,5 \%(n=70)$ não se afastaram de suas atividades laborais enquanto que $37,5 \%(n=42)$ responderam que sim.

Por fim, solicitou-se uma autoavaliação da saúde pessoal, sendo que a maioria, o equivalente a $55,4 \%(n=62)$, avaliou sua saúde como boa, $22,3 \%$ $(n=25)$ avaliou como excelente, $17,9 \%(n=20)$ considerou sua saúde regular, $9 \%$ $(n=4)$ avaliou como muito ruim e $3,6 \%(n=1)$ como ruim.

Quadro 2 - Síntese do perfil dos respondentes

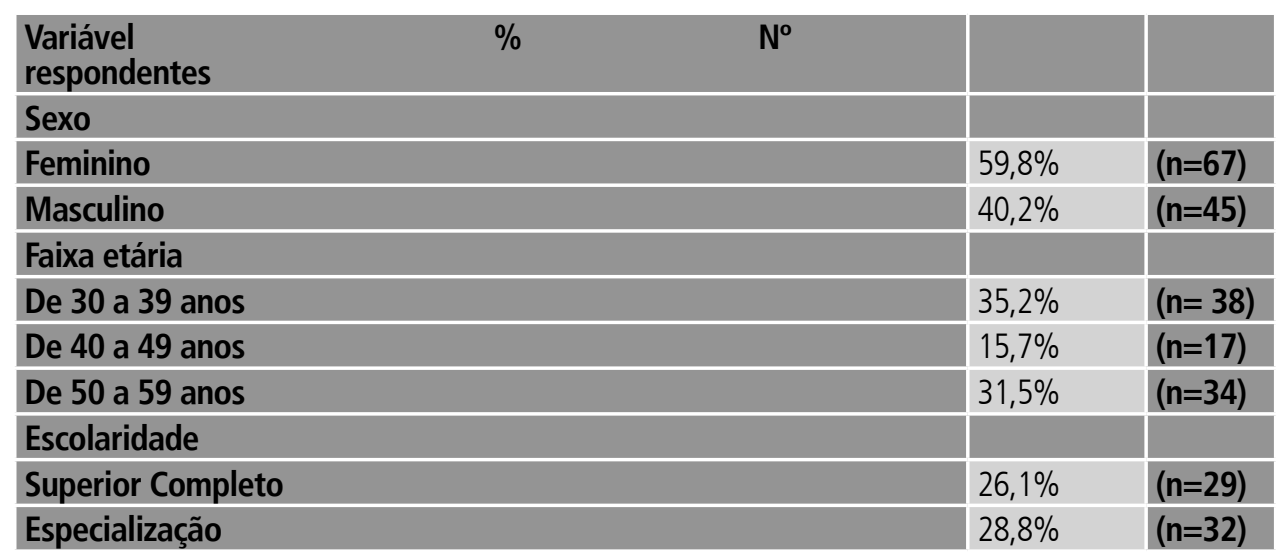




\begin{tabular}{|l|l|l|}
\hline Mestrado & $27 \%$ & $(\mathbf{n}=\mathbf{3 0})$ \\
\hline Tempo de serviço & & \\
\hline Até $\mathbf{3}$ anos & $23,1 \%$ & $(\mathbf{n}=\mathbf{2 5})$ \\
\hline De $\mathbf{4}$ a $\mathbf{9}$ anos & $28,7 \%$ & $(\mathbf{n}=31)$ \\
\hline De $\mathbf{2 0}$ a $\mathbf{2 9}$ anos & $14,8 \%$ & $(\mathbf{n}=\mathbf{1 6})$ \\
\hline Mais de $\mathbf{3 0}$ anos & $21,3 \%$ & $(\mathbf{n}=\mathbf{2 3})$ \\
\hline Cargo de chefia & & \\
\hline Sim & $26,4 \%$ & $(\mathbf{n}=\mathbf{2 9})$ \\
\hline Não & $73,6 \%$ & $(\mathbf{n}=\mathbf{8 1})$ \\
\hline Afastamento por motivo de saúde nos últimos 2 anos & & \\
\hline Sim & $37,5 \%$ & $(\mathbf{n}=\mathbf{4 2})$ \\
\hline Não & $62,5 \%$ & $(\mathbf{n}=\mathbf{7 0})$ \\
\hline Auto-avaliação da saúde & & \\
\hline Ruim & $3,6 \%$ & $(\mathbf{n}=4)$ \\
\hline Muito Ruim & $9 \%$ & $(\mathbf{n}=\mathbf{1})$ \\
\hline Regular & $17,9 \%$ & $\mathbf{( n = 2 0 )}$ \\
\hline Boa & $55,4 \%$ & $\mathbf{( n = 6 2 )}$ \\
\hline Excelente & $22,3 \%$ & $(\mathbf{n}=\mathbf{2 5})$ \\
\hline
\end{tabular}

Fonte: elaborado pelas autoras

\subsection{Análise fatorial da percepção de saúde organizacional}

Para mensurar a percepção com relação à saúde organizacional, o trabalho foi baseado na análise na EPSaO, de Gomide Jr. et al. (1999), revalidada por Gomide Jr. e Fernandes (2008). Para avaliar a confiabilidade da escala, foi realizado o procedimento da análise fatorial exploratória. Para tanto, foi selecionado o método fatorial de análise de componentes principais, o qual considera a variância total e deriva fatores que contêm pequenas proporções de variância única e, em alguns casos, variância de erro (HAIR et al., 2009).

O método utilizado para a extração dos fatores foi o do eixo principal e a rotação Varimax, que maximiza a soma de variâncias de cargas exigidas da matriz fatorial (HAIR et al., 2009), com normalização Kaiser. O índice de Kaiser-Meyer-Olkin - KMO obteve um valor de 0,873 de adequação da amostra e o teste de esfericidade de Barlett foi significativo, indicando a fatorabilidade dos dados.

Em relação às comunalidades, duas variáveis $(I 3=0,433$ e I21 $=484)$ apresentaram valores inferiores a 0,5 , por isso foram extraídas da escala, utilizando-se o critério de comunalidade inferior a 0,50. A escala de Percepção de Saúde Organizacional, agora com 25 itens, foi rotacionada novamente e obteve um valor de KMO de 0,860 e valor do teste de esfericidade de Bartlett de 300, com significância de 0,000 , valores considerados satisfatórios. Contudo, verificou-se que três variáveis apresentavam valores inferiores a 0,5 (I18 = 0,458, 112 $=0,467$ e I17 $=0,410$ ), as quais foram excluídas da escala pelo critério de carga inferior a 0,50. A escala foi rotacionada novamente com 22 itens e obteve um valor de KMO de 0,866 e valor do teste de esfericidade de de Bartlett 231, com significância de 0,000 , valores considerados satisfatórios. 
A estrutura fatorial encontrada foi de seis fatores, constando que o primeiro explica $17,260 \%$ da variância, o segundo explica $16,303 \%$ da variância, o terceiro fator explica $9,800 \%$, o quarto explica $9,163 \%$ da variância, o quinto explica $8,685 \%$ da variância e o sexto explica $8,521 \%$ da variância. O total da variância explicada pelos seis fatores é de $69,733 \%$.

Em seguida, com o propósito de avaliar a confiabilidade da escala, adotou-se o cálculo do coeficiente alfa de Cronbach. Os resultados para cada um dos fatores foram: Fator $1=0,826$, Fator $2=0,879$; Fator $3=0,719$; Fator $4=0,869$; Fator $5=0,704$ e Fator $6=0,698$. Os seis fatores apresentam índices considerados bons, pois, segundo Malhotra (2006), aceitam-se resultados acima de 0,60. Pode-se afirmar que o instrumento possui uma consistência interna satisfatória.

Os fatores receberam as seguintes denominações: Fator 1 - Integração de equipes; Fator 2- Capacidade de flexibilidade da organização; Fator 3 - Relações entre pessoas; Fator 4 - Cooperação entre as pessoas; Fator 5 - Objetivos organizacionais; e Fator 6 - Valor do trabalho. Os fatores e variáveis associadas estão evidenciados no Quadro 3. Ressalta-se que a escala original revalidada por Gomide Jr. e Fernandes (2008) apresenta apenas dois fatores denominados de "Integração de Pessoas e Equipes"; "Flexibilidade e Adaptabilidade a Demandas Externas", divergindo dos achados no presente estudo.

Quadro 3: Fatores extraídos a partir escala de saúde organizacional

\begin{tabular}{|c|c|c|c|}
\hline Questão & & Carga & Variância \\
\hline \multicolumn{4}{|l|}{$\begin{array}{l}\text { Fator 1: Integração de } \\
\text { equipes }(a / p h a=0,826)\end{array}$} \\
\hline 18 & $\begin{array}{l}\text {... as necessidades individuais são levadas em } \\
\text { consideração quando é preciso diagnosticar } \\
\text { problemas organizacionais. }\end{array}$ & 0,690 & 17,260 \\
\hline F26 & ... procura-se continuamente as inovações. & 0,657 & \\
\hline 19 & $\begin{array}{l}\text {... a competição entre equipes é feita de } \\
\text { maneira honesta. }\end{array}$ & 0,640 & \\
\hline 17 & $\begin{array}{l}\text {... procura-se a solução dos problemas de modo } \\
\text { a que todas as pessoas envolvidas possam } \\
\text { opinar. }\end{array}$ & 0,624 & \\
\hline 120 & $\begin{array}{l}\text {... as pessoas aceitam críticas construtivas aos } \\
\text { seus desempenhos no trabalho. }\end{array}$ & 0,612 & \\
\hline F27 & $\begin{array}{l}\text {... as pessoas preparam-se para o futuro, } \\
\text { estando atentas aos } \\
\text { novos métodos de trabalho. }\end{array}$ & 0,603 & \\
\hline $\begin{array}{l}\text { Fator 2: Capacidade } \\
\text { de flexibilidade da } \\
\text { organização (alpha }= \\
0,879 \text { ) }\end{array}$ & & & \\
\hline
\end{tabular}




\begin{tabular}{|c|c|c|c|}
\hline F25 & $\begin{array}{l}\text {... os procedimentos são estabelecidos de modo } \\
\text { a ajudar as } \\
\text { pessoas a serem eficazes no trabalho. }\end{array}$ & 0,813 & 16,303 \\
\hline F24 & $\begin{array}{l}\text {... as políticas são estabelecidas de modo a } \\
\text { ajudar as pessoas a serem eficazes no trabalho. }\end{array}$ & 0,765 & \\
\hline F23 & $\begin{array}{l}\text {... os procedimentos são flexíveis, podendo } \\
\text { adaptar-se } \\
\text { rapidamente às necessidades de mudança. }\end{array}$ & 0,671 & \\
\hline F16 & $\begin{array}{l}\text {... os chefes variam seus estilos de } \\
\text { administração conforme as necessidades das } \\
\text { diferentes situações de trabalho. }\end{array}$ & 0,639 & \\
\hline F22 & $\begin{array}{l}\text {... as políticas são flexíveis, podendo adaptar-se } \\
\text { rapidamente às necessidades de mudança. }\end{array}$ & 0,628 & \\
\hline \multicolumn{4}{|l|}{$\begin{array}{l}\text { Fator 3: Relação entre } \\
\text { pessoas }(a / p h a=0,719)\end{array}$} \\
\hline 14 & $\begin{array}{l}\text {... as pessoas têm acesso às informações } \\
\text { necessárias para } \\
\text { tomar decisões relativas ao trabalho. }\end{array}$ & 0,507 & 9,800 \\
\hline 16 & $\begin{array}{l}\text {... há cooperação entre as pessoas na execução } \\
\text { dos trabalhos. }\end{array}$ & 0,752 & \\
\hline 15 & ... as ações são planejadas em equipe. & 0,582 & \\
\hline 113 & ... as pessoas têm respeito pelas outras. & 0,565 & \\
\hline \multicolumn{4}{|l|}{$\begin{array}{l}\text { Fator 4: Cooperação } \\
\text { entre as pessoas (alpha }= \\
0,869)\end{array}$} \\
\hline 110 & $\begin{array}{l}\text {... as pessoas procuram, espontaneamente, } \\
\text { ajudar seus colegas por meio de sugestões. }\end{array}$ & 0,859 & \multirow[t]{2}{*}{9,163} \\
\hline 111 & $\begin{array}{l}\text {... as pessoas procuram espontaneamente } \\
\text { ajudar seus colegas por meio de ações } \\
\text { concretas. }\end{array}$ & 0,823 & \\
\hline \multicolumn{4}{|l|}{$\begin{array}{l}\text { Fator 5: Objetivos } \\
\text { organizacionais (alpha }= \\
0,704)\end{array}$} \\
\hline 11 & $\begin{array}{l}\text {... as pessoas conhecem os objetivos que a } \\
\text { organização pretende alcançar. }\end{array}$ & 0,834 & \multirow[t]{2}{*}{8,685} \\
\hline 12 & $\begin{array}{l}\text {... as pessoas trabalham unidas para que a } \\
\text { organização alcance seus objetivos. }\end{array}$ & 0,734 & \\
\hline \multicolumn{4}{|l|}{$\begin{array}{l}\text { Fator 6: Valor do trabalho } \\
(\text { alpha }=0,698)\end{array}$} \\
\hline I14 & $\begin{array}{l}\text {... as pessoas encaram seus trabalhos como } \\
\text { algo importante. }\end{array}$ & 0,805 & \multirow[t]{3}{*}{8,521} \\
\hline I15 & $\begin{array}{l}\text {... as pessoas encaram seus trabalhos como } \\
\text { algo prazeroso. }\end{array}$ & 0,565 & \\
\hline 119 & $\begin{array}{l}\text {... as pessoas sabem o que é importante } \\
\text { para a organização. }\end{array}$ & 0,517 & \\
\hline
\end{tabular}

Fonte: elaborado pelas autoras.

O primeiro fator, denominado "Integração de equipes", apresentou variância explicada de $17,260 \%$, alpha igual a 0,826 e ficou composto por seis variáveis, duas relativas à consideração das necessidades individuais e opinião de todos envolvidos na solução de problemas; uma referente à aceitação de 
críticas construtivas; outra sobre competição entre equipes; duas se referem à inovação no trabalho que, na escala original, pertenciam à dimensão Flexibilidade e Adaptabilidade a Demandas Externas. Baltzer et al. (2011) defendem que a gestão participativa contribui com a saúde institucional através da participação de todos os envolvidos no processo de trabalho, à medida que Faria et al. (2017) complementam que a construção de espaços coletivos, nos quais todos os integrantes tenham a oportunidade de expressar suas visões e opiniões, fortalece o processo educativo no cenário universitário.

No segundo fator, chamado de "Capacidade de flexibilidade da organização", com variância de 16,303 e alpha igual a 0,879, composto por cinco variáveis, relativas à capacidade de organização ser flexível, possibilitando que as pessoas sejam eficazes no trabalho, bem como estejam preparadas quando houver necessidade de mudanças e ainda quanto à capacidade da chefia ser flexível e adaptável às situações de trabalho. Acrescenta-se que esse fator reuniu cinco das sete variáveis que, na escala proposta por Gomide Jr. et al. (1999), estavam concentradas no fator "Flexibilidade e Adaptabilidade a Demandas Externas". Considerando que a maneira como as organizações são estruturadas e operam podem gerar impactos na saúde e no desempenho dos funcionários, deve-se enfatizar uma interação dinâmica entre fatores individuais e organizacionais (DEJOY e WILSON, 2003).

Quanto ao terceiro fator, "Relação entre pessoas", percebe-se que as quatro variáveis nele inseridas se referem ao envolvimento interpessoal, mais especificamente se há respeito entre as pessoas, trabalho em equipe, cooperação na execução dos trabalhos e acesso a informações necessárias para a tomada de decisões. Já o quarto fator, "Cooperação entre pessoas", com alpha igual a 0,869, apresenta duas variáveis que visam verificar se existe ajuda entre os colegas por meio de sugestões e ações concretas. As relações interpessoais são essenciais para os servidores, desde os mais novos aos mais antigos, ainda mais no contexto do serviço público que devido à continuidade da carreira é possível cultivar amizades e bons relacionamentos. Assim, as relações são um elemento estratégico na organização do ambiente de trabalho, pois junto a fatores como: a transparência, participação na gestão, respeito às pessoas, valorização e reconhecimento, promovem benefícios à saúde dos trabalhadores e consequentemente à saúde da organização (FARIA et al., 2017).

O quinto fator, com alpha de 0,704 , variância igual a 8,685\%, abrange duas variáveis referentes aos objetivos da organização. Para Filho (2015), possibilitar organizações comprometidas com sua missão pública garante qualidade na prestação de serviços públicos, além de ser uma ferramenta eficaz no cuidado da saúde de seus servidores. Importante mencionar que Vicentini et al. (2010) encontraram um fator com as mesmas variáveis quando estudaram a saúde organizacional como influenciadora do estresse do trabalho. Ao verificar a fatorabilidade da EPSaO, os autores, inicialmente, também encontraram seis fatores, contudo, como o alpha foi inferior ao valor mínimo sugerido, op- 
taram pela eliminação desse fator, o qual haviam denominado como "Objetivos organizacionais".

Já o sexto fator, "Valor do trabalho", apresenta alpha igual a 0,698, variância explicável de 8,521\% e compreende três variáveis atinentes à importância do trabalho para as pessoas e para a organização. Conforme Oleto et al. (2013, p. 570), "O trabalho é rico de sentido individual e social, e vai além de ser um meio de produção da vida de cada um ao prover subsistência, mas cria sentidos existenciais e contribui para a estruturação da identidade e da subjetividade". Assim, a importância do trabalho na vida das pessoas está além da necessidade de manutenção financeira, tendo em vista que também pode ser um meio de realização pessoal e de promoção da dignidade da pessoa humana.

Considerando que o contexto organizacional, as relações interpessoais, a rigidez das normas e regras das organizações podem ser fonte de sofrimento e adoecimento (OLETO et al., 2013) comprometendo a saúde pessoal e organizacional, importante que as organizações se desenvolvam de maneira que se reconheça o potencial da sua força de trabalho, valorizando os vínculos dos indivíduos com as organizações.

Para Faria et al. (2017), as universidades precisam proporcionar ambientes de trabalho saudáveis e estimulantes para potencializar a identidade das pessoas com a instituição, ou seja, gerar sensação de pertencimento. Ainda, refere que a melhoria dos processos de trabalho, por meio da participação de todos envolvidos, amplia a saúde institucional e, por consequência, a qualidade dos serviços prestados, "dentro da lógica dos valores públicos de equidade e justiça, num contexto de aprendizado da democracia" (FARIA et al., 2017, p. 543).

\section{Considerações finais}

O estudo da temática saúde organizacional é relevante para as organizações, pois se trata de um assunto que contribui para o sucesso de uma organização e, ao mesmo tempo, cuida da saúde e do bem-estar dos funcionários. Com base na análise bibliográfica realizada, pode-se observar a complexidade que envolve o assunto, tendo em vista que não possui critérios bem definidos para que se possa avaliar se uma instituição possui ou não saúde organizacional. Além de não apresentar um conceito consolidado, os elementos/fatores que devem ser observados para se verificar a saúde de uma organização variam conforme entendimento de cada pesquisador.

Os resultados dos dados e informações coletados permitiram a identificação do perfil da maioria dos respondentes: predominância do sexo feminino, faixa etária média entre 30 e 39 anos. No que tange à escolaridade, a maior parte são servidores com especialização, o que reflete o alto nível dos servidores no que diz respeito ao nível de instrução. Quanto ao tempo de serviço, em sua maioria, estão de 4 a 9 anos na instituição.

No modelo de escala original, de Gomide Jr. et al (1999), as variáveis 
são dispostas em dois fatores, o que não se confirma nessa pesquisa, tendo em vista que foram encontrados seis fatores, com variância total explicada de $69,733 \%$. Os resultados sugerem a necessidade de maiores investigações quanto à utilização de apenas dois fatores para medir a percepção de saúde organizacional. Vicentini et al. (2010), ao estudarem a saúde organizacional como influenciadora do stress no trabalho, obtiveram $62,42 \%$ de variância total explicada para os seis fatores inicialmente formados, no entanto, dois fatores foram excluídos após a verificação da confiabilidade, permanecendo quatro fatores no modelo final.

Estudos sobre saúde organizacional ainda são poucos e demandam mais análises e desenvolvimento, fator que inviabilizou uma comparação mais aprofundada dos resultados encontrados nessa pesquisa. Assim, considera-se que o estudo contribuiu no sentido de ter investigado o incipiente constructo saúde organizacional tanto nas pesquisas de âmbito da universidade em análise, quanto para a gestão de organizações públicas no Brasil. Por fim, a disposição dos dados resultantes dessa pesquisa poderá ajudar os gestores que têm a competência de elaborar e implementar políticas de gestão de pessoas a repensarem os aspectos que interferem na saúde da instituição.

As limitações desse trabalho referem-se ao fato de que a amostra foi formada basicamente por técnicos administrativos em educação. Sugere-se que outras pesquisas sejam realizadas com este modelo para um número maior de servidores, incluindo os docentes, ainda investiguem a correlação do constructo com aspectos do contexto socio-organizacional, tais como: envolvimento com o trabalho, comprometimento organizacional e o bem-estar dos servidores.

\section{Referências}

BALTZER, M.; WESTERLUND, H.; BACKHANS, M.; MELINDER, K. (2011) Involvement and structure: a qualitative study of organizational change and sickness absence among women in the public sector in Sweden. BMC public health. London, v. 11, p. 318, May.

BENNIS, W.G. (1962) Towards a truly scientific management: The concept of organizational health. General Systems Yearbook, 7, 269-282.

CARNEIRO, S. A. M. (2006). Saúde do trabalhador público: questão para a gestão de pessoas - a experiência na Prefeitura de São Paulo. Revista do Serviço Público - Brasília 57 (1): 23-49 Jan/Mar.

CASSANDRE, M. P. (2011) A Saúde de Docentes de Pós-graduação em Universidades Públicas: Os Danos Causados pelas Imposições do Processo Avaliativo. Revista Mal-estar e subjetividade - Fortaleza - vol. XI $N^{\circ} 2$ - p. 779 - 816 - jun.

COX, T.; HOWARTH, S. (1990) Organizational health, culture and helping. Work and Stress, London, v. 4, n. 2, p. 107-110.

CRESWELL, J. W. (2010) Projeto de pesquisa: método qualitativo, quantitativo e misto. - 3. ed. - Porto Alegre : Artmed.

DEJOY, D.M.; WILSON, M.G. (2003) Organizational Health Promotion: Broadening the Horizon of Workplace Health Promotion. American Journal of Health Promotion, 17 (5), p. 337-341. 
A SAÚDE ORGANIZACIONAL: ESTUDO DE CAMPO EM UMA INSTITUIÇÃO DE ENSINO SUPERIOR

FARIA, R. M. O.; LEITE, I. C. G.; SILVA, G. A. (2017) O sentido da relação trabalho e saúde para os assistentes em administração de uma universidade pública federal no Estado de Minas Gerais. Revista de Saúde Coletiva, Rio de Janeiro, 27 [ 3 ]: 541-559. DOI: http://dx.doi.org/10.1590/S010373312017000300009 .

FERNANDES, M. N.; GOMIDE JR., S.; OLIVEIRA, A. F. (2011). Saúde Organizacional: uma Proposta de Modelo de Análise. Revista Psicologia: Organizações e Trabalho, 11, 1, jan-jun 2011, p. 54-65.

FILHO, J. M. J. (2015) Engajamento no trabalho, impedimentos organizacionais e adoecer: a contribuição da Ergonomia da Atividade no setor público brasileiro. Rev. bras. Saúde ocup., São Paulo, 40 (131): 98-108.

GEORGOPOULOS, B.; TANNENBAUM, A.S. (1957). A study of organizational effectiveness. American Sociological Review, Washington, 22, 534-540.

GIL, A. C. (2009) Como elaborar projetos de pesquisa. $4^{a}$ ed. - 12. São Paulo : Atlas.

GOMIDE JR., S.; FERNANDES, M.N. (2008) Saúde Organizacional. IN: SIQUEIRA, M.M.M. Medidas do comportamento organizacional: ferramentas de diagnóstico e de gestão / Mirlene Maria Siqueira (Org.) ; Álvaro Tamayo ... [et al.]. -Dados eletrônicos. - Porto Alegre : Artmed.

GOMIDE Jr., S.; MICHELETTO, M. R. D. (2013) Saúde organizacional: inserção, valorização e sua pertinência para a área da saúde do trabalhador. R. Laborativa. v. 2, n. 1, p. 66-70, abr./2013. http://ojs.unesp.br/index. php/ rlaborativa.

GOMIDE JR., S.; MOURA, O.I.; CUNHA, W.B.; SOUSA, W.M.V. (1999) Explorando o conceito de Saúde Organizacional: construção e validação de um instrumento de medida para o ambiente brasileiro. In: SOCIEDADE BRASILEIRA DE PSICOLOGIA, 29., Campinas. Resumos.... p.43.

HAIR Jr., J.F.; BABIN, B.; MONEY, A.H.; SAMOUEL, P. (2009) Fundamentos de métodos de pesquisa em administração. 6 ed. Porto Alegre: Bookman.

HART, P.M.; COOPER, C.L. (2001). Occupational stress: Toward a more integrated framework. In N. Anderson, D.S. Ones, H.K. Sinangil, \& C. Viswesvaran (Eds), Handbook of Industrial, Work and Organizational Psychology: Vol. 2. Personnel psychology (pp. 93-114). London, UK: SAGE Publications.

JAFFE, D. T. (1995) The healthy company: research paradigms for personal and organizational health.

KATZ, D.; KANH, R.L. (1966) The social psychology of organizations. New York: Wiley.

LOUREIRO, T.; MENDES, G. H. S.; SILVA, E. P. (2018) Estigma, invisibilidade e intensificação do trabalho: estratégias de enfrentamento do sofrimento pelos assistentes em administração Trab. Educ. Saúde, Rio de Janeiro, v. 16 n. 2, p. 703-728, maio/ago. DOI: http://dx.doi.org/10.1590/1981-7746-sol00111

MACINTOSH, R.; MACLEAN, D.; \& BURNS, H. (2007) Health in organization: Towards a process-based view. Journal of Management Studies, 44(2), 206-221.

MALHOTRA, N. K. (2006) Pesquisa de marketing: uma orientação aplicada. 3. ed. Porto Alegre: Bookman.

MELLO, F.A.F. (1978) Desenvolvimento das organizações: uma opção integradora. Rio de Janeiro: Livros Técnicos e Científicos.

NASCIMENTO, J. O.; GOMIDE JÚNIOR, S. (2008) Percepções de saúde e efetividade organizacionais: construção, validação e discriminação das medidas dos constructos. IX Encontro Interno \& XIII Seminário de Iniciação Científica. UFU - 30 anos.

Oleto, A. F., MELO, M. C. O. L. \& LOPES, A. L. M. L. (2013) Análise Bibliométrica da Produção Sobre Prazer e Sofrimento no Trabalho nos Encontros da Associação Nacional de Pós-Graduação em Administração (2000-2010). Psicologia: Ciência e profissão, 33 (1), 60-73.

QUICK, J.C.; MACIK-FREY, M.; \& COOPER, C.L. (2007) Managerial dimensions of organizational health: The healthy leader at work. Journal of Management Studies, 44(2), 189-205.

REDDIN, W. J. (1970) Effectiveness Managerial. McGraw-Hill: Book Company.

RIBEIRO, C. V. S. (2012) O trabalho do técnico-administrativo em instituições federais de ensino superior: análise do cotidiano e implicações na saúde. R. Pal. Públ. São Luis MA Número Especial, p. 423432.

SAUTER, S.; LIM, S.; \& MURPHY, L. (1996) Organizational health: A new paradigm for occupational stress research at NIOSH. (Japanese Journal of) Occupational Mental Health, 4, 248-254. 
SCHEIN, E. H. (1965) Organizational Psychology. Foundations of Modern Psychology series. EnglewoodCliffs, NJ: Prentice-Hall.

SHOAF, C.; GENAIDY, A.; KARWOWSKI, W.; HUANG, S.H. (2004) Improving Performance and Quality of Working Life: a model for organizational Health Assessment in Emerging Enterprises. Human Factors and Ergonomics in Manufacturing, 14 (1), p.81-95.

SINGH, A.; JHA, S. (2017) Scale Development of Organizational Health Construct. Global Business Review 19(2) 357-375. http://journals.sagepub.com/home/gbr.

VISENTINI et al. (2010) Empresa doente, funcionário estressado: analisando a saúde organizacional como influenciadora do stress no trabalho. Revista de Ciências da Administração, v. 12, n. 26, p. 189-220.

WILSON, M.G.; DEJOY, D.M.; VANDENBERG, R.J.; RICHARDSON, H.A.; MCGRATH, A.L. (2004). Work characteristics and employee health and well-being: test of a model of healthy work organization. Journal of Occupational and Organizational Psychology, 77 (4). 